\title{
DCS Technology-Based Design of Electrical Control System for Thermal Power Plant
}

\author{
Kepei $\mathrm{Li}^{1^{*}}$ and Gia Vani ${ }^{2}$ \\ ${ }^{I}$ Department of Electrical Engineering, Dezhou Vocational and Technical College, Dezhou 253000, China \\ ${ }^{2}$ University Science Instrumentation Centre, Gulbarga University, Gulbarga
}

\begin{abstract}
Distributed Control System (DCS) has been widely recognized in the field of modern thermal power thermal control, and has significant effects in the application process. Today, the monitoring of electrical quantities is included into the DCS system, thus replacing the traditional "one to one" manual mode of operation in electrical control. It is to further improve the main works of the station automation and control systems. Combined with the actual project needs, this study focuses on the components of electrical control included in DCS system, the implementation of software and hardware features, and the measures to improve reliability. In addition, useful discussions have been made for some technical problems. In the DCS system, the electrical control inclusion is not just a perfection thermal power plant electrical control system, but a big improvement as well. In addition, it is a new attempt. At present, most of the electrical control parts of control system included into the DCS system with the manual operation are basically cancelled, but there are some problems, for example many of the designs do not match with the original operating habits, etc., which will have a certain impact on the tradition. In the transformation process of system, some of the designs are good but cannot be achieved currently. They still need further research and improvement, for example how can the electrical control of system enter into the DCS in a better manner, how can the display of light texts upon failure or abnormal time weaken electric signals in a more comprehensive and rapid manner, what are the new requirements for anti-interference, and other sequential control functions to be improved and perfected.
\end{abstract}

Keywords: DCS technology, electrical control system, electrical control, system reliability, thermal power plant, industry.

\section{INTRODUCTION}

In order to include the electrical control into DCS, we must fully consider the difference between the electrical system and the thermal industrial control system and the specific needs of electrical control system on consisting of hardware and software as well as the functional logic implementation, so as to ensure high reliability and security of the system. Due to its less controlled objects and much lower operating frequency, electrical system is a little simpler than the thermal system [1]. However, the operation of the electrical equipment has very high reliability requirements, and any negligence and mistakes can cause very serious consequences. In addition, the electrical system requires a certain degree of independence in operation, for example plant system must be put into operation first. Electrical relaying protection and automatic safety devices not only have high reliability requirements, but also they are featured by fast response and precise action parameters as well. For example, rapid protection's export time is usually $30 \sim 40 \mathrm{~ms}$, automatic synchronization switching has the advance timing of $<100$, and they have precise phase angle and slip frequency measurements, etc. The electrical equipment inter-chain conditions are

\footnotetext{
*Address correspondence to this author at Department of Electrical Engineering, Dezhou Vocational and Technical College, Dezhou 253000, China; Tel: +8613455403892; E-mail: likepei@126.com
}

relatively simple, but the certain component failure may result in large affected area. In addition, the operation mechanism of the electrical device itself is complex, and this part of the chain conditions will be independent of the DCS system to play a role. In addition, the operation of electrical system not only shall meet the requirements of the operation of this unit, it must also obey the grid scheduling commands.

\section{INTRODUCTION TO ELECTRICAL SYSTEM IN- CLUDED INTO THE DCS}

The work breakdown structure of F Power Plant DCS project, contents included and main participants in each work package are shown in Table 1: With the continuous development of digital protective relay test technology, test has gradually transformed from the traditional manual testing into automatic test. It is a major development trend. The introduction of automated tools for automated testing in the testing process is a very efficient and practical method [1]. Automated testing test is featured by repeatability and ensures traceability of test works; automated test can make use of the advantages of various testing methods of its system, expand test coverage, and ensure the integrity of test [2]. In the installation automated test, the test task is automatically generated, the test process is performed automatically, the test results are automatically compared and test report is automatically generated. The manual interference is minimized in the test, excluding testing randomness and repeated test, 
Table 1. Contents included and participants in each stage of project.

\begin{tabular}{|c|c|c|c|}
\hline $\begin{array}{l}\text { Project Phas- } \\
\text { es }\end{array}$ & Work Breakdown & Contents to be Determined & Main Participants \\
\hline \multirow[t]{2}{*}{1 Kick-off } & $\begin{array}{l}\text { 1.1 Project kick-off } \\
\text { meeting }\end{array}$ & $\begin{array}{l}\text { Power status communication; understanding of contracts and } \\
\text { technical clarification; determine the organization method and } \\
\text { timing of the project. }\end{array}$ & $\begin{array}{l}\text { Owner, design institute, project imple- } \\
\text { mentation unit }\end{array}$ \\
\hline & $\begin{array}{l}1.2 \text { Allocation of re- } \\
\text { sponsibilities }\end{array}$ & Responsibilities of project members, project resource allocation. & Project implementation unit \\
\hline \multirow[t]{3}{*}{2 Design } & 2.1 Overall design & DCS control scope, control depth and control method. & $\begin{array}{l}\text { Owner, design institute, project imple- } \\
\text { mentation unit }\end{array}$ \\
\hline & 2.2 Preliminary design & $\begin{array}{l}\text { Preliminary design of hardware, software preliminary design, } \\
\text { human-computer interface design. }\end{array}$ & Project implementation unit \\
\hline & 2.3 Detailed design & Function realization method, optimal control design issuance. & Project implementation unit \\
\hline \multirow[t]{2}{*}{3 Installation } & $\begin{array}{l}\text { 3.1 Hardware installa- } \\
\text { tion }\end{array}$ & $\begin{array}{l}\text { Network topology structure, the type and number of network } \\
\text { components, facility security, etc. }\end{array}$ & Project implementation unit \\
\hline & $\begin{array}{l}\text { 3.2 Software installa- } \\
\text { tion }\end{array}$ & $\begin{array}{l}\text { What software shall server, DCS workstation, operator station, } \\
\text { engineer station and other equipment install. }\end{array}$ & Project implementation unit \\
\hline \multirow[t]{3}{*}{4 Debugging } & $\begin{array}{l}\text { 4.1 Hardware debug- } \\
\text { ging }\end{array}$ & $\begin{array}{l}\text { Is the equipment operating properly, the effect of the various } \\
\text { hardware joint commissioning. }\end{array}$ & Project implementation unit \\
\hline & 4.2 Software debugging & $\begin{array}{l}\text { Has the design features of each software been completed, soft- } \\
\text { ware joint test results. }\end{array}$ & Project implementation unit \\
\hline & 4.3 Joint test & $\begin{array}{l}\text { Is the joint commissioning of hardware and software operating } \\
\text { properly, do they have the project acceptance conditions. }\end{array}$ & Project implementation unit \\
\hline
\end{tabular}

which can greatly improve testing efficiency and credibility. Automated testing technology has received a lot of research and practice at home and abroad, and they have made great progress [2]. But the current relaying automatic test still has some deficiencies, so we need to further study and improve. They are mainly reflected in the following aspects:

(1) The current automatic test system can only achieve automated testing of single protective equipment, and there is the lack of a solution at system for two or more equipment, such as the solution providing concurrent automated testing of all protective devices on a single line.

(2) The system is not strongly universal. Due to a wide range of protection and testing procedures that vary in different regions, it is impossible to generate state sequence for common use in testing for some kind of protective equipment, in a one-off manner. In addition, in system testing process, the information is not saved or is saved incompletely. As a result, once there are problems during testing, investigation will be very troublesome [3].

Most existing automatic test systems have a protocol analysis module that uses the 103,104 and other protocols and has information exchange with the device under test, complete unit valuing, wave plate control and recording data acquisition; tester control module issue orders to the tester of completing simulation in power fault process; humancomputer interactive interface mainly completes the test case editing, information display during the execution of test task, test report display, and other functions. The entire system generally uses automatic test software installed with a PC, and a tester to add amount to the device under test. Through network connection, it can enable the testing of relay protection device under test. The contents included and participants of each stage of project are shown in Table $\mathbf{1}$.

\section{INTRODUCTION TO NORMALLY CLOSED CON- TACTS}

In order to reflect the operational status of electrical switches, what was used in the past was the normally open switch contacts introduced to DCS. The closure of normally open contact means switch is in closing state, and the openness of normally open contact means the switch is in the brake off condition. However, if it is found in actual operation, only one pair of normally open contact to reflect the state of switch, will be extremely unreliable. Fig. (1) is a logic judgment of the electrical switching accident and tripping.

Reuse of test case is difficult, for different models of the same kind of device, test cases for the original model require a lot of changes, and even the use case shall be re-edited. In automated testing, use case editing is relatively the most time-consuming, and the use case cannot be reused well, which greatly limits the effectiveness of automated testing. There is lack of use case management. Test case is the core 


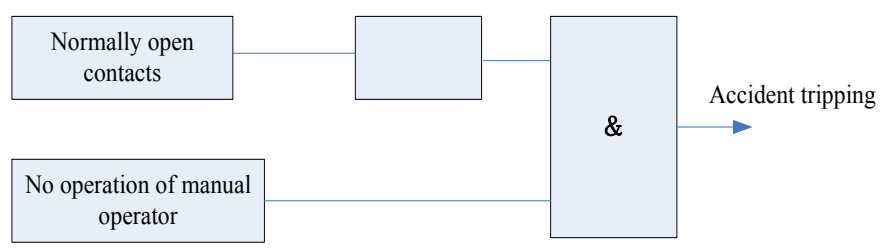

Fig. (1). Accident tripping logics of electrical switch.

of the entire test, and the necessary use case management function is essential; for example, at the end of test for a project or the cross-test between projects, the saving of use case and configuration environment depends on manual work. When performing the same usage in the next time, manual loading of the use cases and configuration test environment are required, seriously affecting the efficiency of test. There is lack of normative detection function for test [4]. The normative feature of test cases directly determine the success or failure of the subsequent automated testing; for example, in testing with the use of previous automatic system, because the tester manually changes one test case, the use case is malformed, resulting in the automatic execution failure of entire project, so the system fault tolerance is poor.

In view of the above problems facing relay automatic test system, and fully taking into account various factors, such as existing software technology, tester technology, and IEC61850 communication protocol, etc. this study proposes a set of practical automatic test system design program. The program can effectively solve the problems of multi-device testing, system versatility and testing process information saving, the test case reuse problems, the test case management problems, the test case legality testing and a series of complex problems. Fig. (1) shows accident tripping logics of electrical switch.

\section{CONTROL LOOP OF REAL-TIME MONITORING OF ELECTRICAL SWITCH}

For installation of additional HWJ, TwJ relay in the original electrical control circuit, on the one hand it can monitor the integrity of the control power supply, on the other hand it will monitor the closing and tripping circuit integrity. Specific wiring is shown in Fig. (2). In DCS screen, the red light HD is to reflect HWJ state, the green light LD is to reflect TwJ state, which is consistent with many years of operating experience of operating personnel. On the other hand, when HWJ, TWJ are de-energized, the light texts and sound alarm can be generated to notify the operator to deal with the problems promptly [5].

Criteria inspection; there are four criteria: protection exit criterion, tester criterion, return coefficient criterion, and alarm remote signaling criterion. These four criteria are used for the judgment of implementation, with success or failure results. If it is successful, it will no longer analyze generally. If the use case fails, tester shall analyze the reasons of failure and position the problems.

Clean-up work; after the test is completed, clean up the site and make preparation for the test of the next use case. Upon execution completion of one test case, the status needs to be restored before the relay test is performed so as to en- sure that it does not affect the execution of the next use case. Different test cases are designed for different test point devices, so it is necessary to make sure the independence of the use case execution.

In the protection logic test, the complete implementation process of one protection use case is used to introduce implementation process of protection logic test. Click to start the test, and then the program will first start the abnormality checking function, including whether to choose the device under test, whether the device selected is online, whether to establish normal communications with the selected device. Then load test case (test case is designed in accordance with device test point, with one use case corresponding to one or more test points) to test the device under test. Three processes of test are in accordance with the testing process in front of us: condition preparation, criterion check, and cleaning work (after test completion). After a test case is completely executed, there will be a few seconds delay to start the execution of the next use case. Cycling in the proper order, the test will come to an end until the use cases to be executed are fully executed.

As mentioned in the previous functional analysis section, the type of tests that are mainly supported by this test system include protection logic testing, plate testing, valuation testing, remote testing, telemetry testing, remote testing. Among them, protection logic test is the core module of system, because the testing of protection device is mainly to test its protection logic. Test of plate and fixed values is the basic test, and these three types of tests account for more than $80 \%$ in test of protective device, and the test logic of plate and fixed value is very similar. Due to the limited length, only the design and implementation of two test types, i.e. protection logic test and plate test are detailed below. Other types are temporarily not described in detail. Fig. (2) shows the HWJ and TWJ elementary wiring diagram.

Many electrical switch auxiliary contacts are leakagestyle, which makes them easy to rust; but DCS system has input voltage of $48 \mathrm{~V}$, and it is difficult to ensure contact reliability. So in our company's 5 machine DCS transformation, we have taken a method to convert the switch contact via relay and then convert it into the DCS as shown in Fig. (3).

As it can be seen from Fig. (3), the isolation cabinet uses $220 \mathrm{~V}$ AC power supply voltage, and the practice has proved that this design method has the following flaws in the application:

(1) Interference problem. Some DC switch contacts use the same cable with other DC circuit, causing serious interference on DC circuit, and the maximum voltage of $100 \mathrm{v} \mathrm{AC}$ can be induced. 


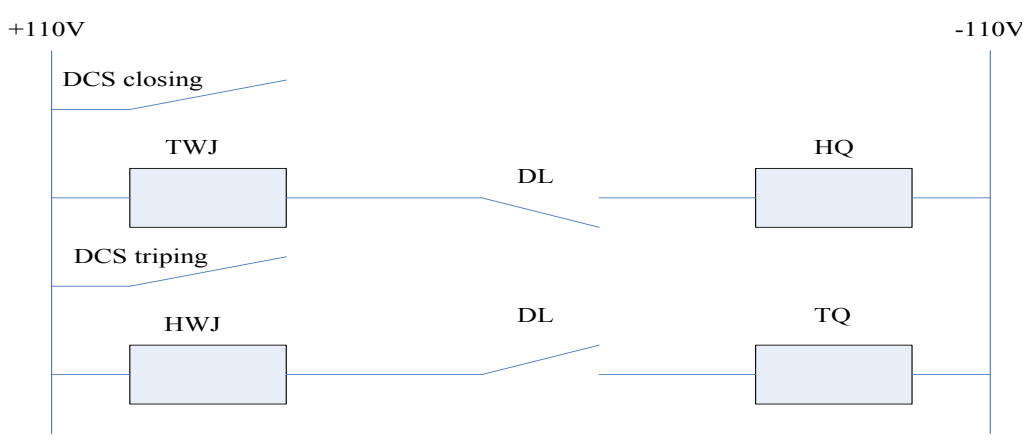

Fig. (2). HWJ and TWJ elementary wiring diagram.

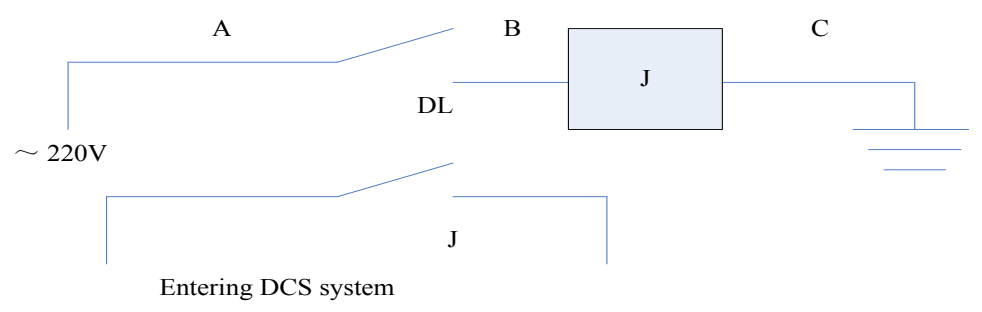

Fig. (3). Isolation cabinet function diagram.

(2) It may lead to the AC power short circuit. In actual operation, the switch contacts may have grounding phenomenon. As shown in Fig. (3), if Point A is grounded, but the processing flow is complex. Because the plate is unlike the fixed value, for which the test system can obtain all valuation information by directly communicating with the device under test. Plate needs the help of monitoring background in order to obtain the plate information of device under test. Plate state is only in two types: casting and returning, but there are a wide variety of combinations.

(3) It may lead to DCS erroneous determination. During the actual wiring, if Point $\mathrm{A}$ is changed into ground connection, Point $\mathrm{C}$ is changed into $\mathrm{AC} 220 \mathrm{~V}$, then, when the switch contact has grounding at Point $\mathrm{B}$, the relay inside the isolation cabinet $\mathrm{J}$ will malfunction, causing erroneous determination of the internal DCS that this switch contact is closed.

In view of the above-mentioned phenomenon of insecurity, in the DCS transformation of the company's other units, we change the power supply of isolation cabinet into the more reliable DC power supply.

\section{SCOPE OF ELECTRICAL SYSTEM INCLUDED INTO DCS}

The transformation is performed on the 5th unit. According to the 5th unit characteristics of using generator transformer circuit group wiring and unit control, the transformation work includes all electrical systems of this unit, including generating and transformer group, electricity lines and plant use electricity as well as the unit public electrical systems into the DCS monitoring.

According to the primary device to be protected, the relay protection devices can be simply divided into line protection, bus protection, transformer protection, and generator transformer unit protection. The main function of circuit protection device is to have protection functions necessary for a variety of transmission line failures, which are independent and complete; main function of bus bar protection device is to operate correctly in the event of various faults in the bus zone; main transformer protection device has the function to have an independent and complete reflection to various failures and abnormal state of transformer, and take action on the tripping or to give signals; generator transformer unit protection has the main function to have independent and full completion protection functions required for protecting equipment [6].

Detecting the core function of relay protection device can realize logical test for various types of protection. For example, for line protection type, there are mainly fiber differential protection, high frequency protection, distance protection, zero sequence protection, reclosing, etc.; the main type of bus protection includes differential protection, bus-couple protection, breaker failure protection; the main types of transformer protection are ratio differential protection, overexcitation protection, re-pressed directional overcurrent protection, zero sequence directional protection, impedance protection; the main types of protection for generator and transformer include generator ratio braking-type fully differential protection, generator interterm protection, split-phase transverse differential protection, stator ground fault protection, low excitation, loss protection, stall protection, reverse power protection, over-excitation protection, low voltage memory overcurrent protection, negative sequence inversetime overcurrent protection, rotor overload protection, overload protection symmetrical impedance protection, over frequency protection, low frequency protection, generator power protection for misconnecting, the downtime stator ground fault protection, electrical over speed protection, etc. Work breakdown structure of F Power Plant DCS program is shown in Fig. (4). 


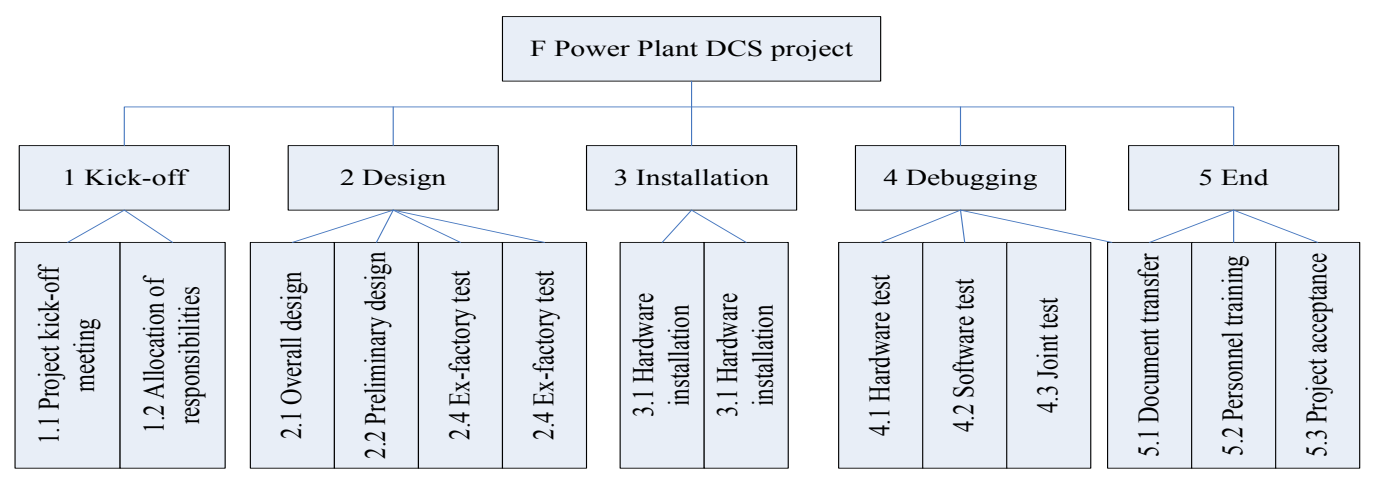

Fig. (4). Work Breakdown Structure of F Power Plant DCS Project.

Protection logic is the core function of protection devices, so protection logical test is the core of the entire automatic test system and its implementation process is also the most representative one. Protection logic testing process can be divided into three phases: preparation conditions, the criterion inspection, cleaning work (after test completion).

Condition preparation and test preparation works mean the pre-cast plate of protection logic test, writing fixed value, passing the file of tester to client side of tester, and starting the tester output and other operations. For taking the protection of functional test for example, the main purpose is to test the protection logic, so the success of the condition preparation shall be ensured (we need to make sure the success because the preparation conditions may fail because device is busy preparing recorded wave). Several preparation conditions are used to ensure its success. This study is designed to have the maximum preparation number of 20 . More than 20 times repeat without success will be regarded as a test failure, and the tester needs to analyze the reasons for failure. In the process of condition preparation, the operation of plate and fixed value has four states in the operation task list, including pending, execution, completion and disintegrating (and adding to the restoring list) [7].

\section{CONCLUSION}

This study mainly describes the implementation of including electrical control into the DCS system components, software and hardware features and measures to improve the reliability, and we make useful discussions on some technical problems. In the DCS system, the electrical control inclusion is not just a perfection thermal power plant electrical control system, but a big improvement as well. In addition, it is a new attempt. At present, most of the electrical control parts of control system are included into the DCS system with the manual operation basically cancelled, but there are some problems, for example many of the designs do not match with the original operating habits, etc., which will have a certain impact on the tradition. In the transformation process of system, some of the designs are good but cannot be achieved currently. They still need further research and improvement, for example how can the electrical control of system enter into the DCS in a better manner, how can the display of light texts upon failure or abnormal time weaken electric signals in a more comprehensive and rapid manner, what are the new requirements for anti-interference, and other sequential control functions to be improved and perfected.

\section{CONFLICT OF INTEREST}

The authors confirm that this article content has no conflict of interest.

\section{ACKNOWLEDGEMENTS}

Declared none.

\section{REFERENCES}

[1] W. Li and G. Liang, "Transformation and application of DCSbased electrical control system in medium and large sized thermal power plant", Electrical Application, vol. 5, no. 1, pp. 26-30, 2012.

[2] S. H. Jiao, J. Li, W. Li, and Y. Liu, "The achieving pattern of electrical control system in large-sized thermal power plant", Power System Automation, vol. 15, no. 8, pp. 236-239, 2006.

[3] M. Zhang, H. Q. Zhang, and X. H. Du, "Description and security analysis of PKI-based SSL protocol", Micro Computer Information, vol. 12, no. 3, pp. 51-53, 2006.

[4] C. L. Xiao and Y. Q. Liang, Web Services Technology Architecture and Application. Beijing: Electronic Industry Press, 2007.

[5] W. X. Ma, H. S. Zhong, and T. J. Yan, "Research on application of industrial Ethernet-based electrical automation system in thermal power plant", Journal of Software, vol. 18, no. 10, pp. 2551-2563, 2007.

[6] Q. Y. Wang, R. E. Li, and T. G. Li, "Design realization of unified user management and identity authentication services", Mechanical \& Electrical Engineering Technology, vol. 21, no.3, pp. 7-12, 2009.

[7] M. C. Zheng, "Discussion over the applications of DCS system in electrical engineering of thermal power plant", Hunan Hydro \& Power, vol. 20, no. 6, pp. 55-59, 2011.

Received: July 28, 2014

Revised: November 09, 2014

Accepted: November 27, 2014

(C) Li and Vani; Licensee Bentham Open.

This is an open access article licensed under the terms of the Creative Commons Attribution Non-Commercial License (http://creativecommons.org/licenses/by-nc/4.0/) which permits unrestricted, non-commercial use, distribution and reproduction in any medium, provided the work is properly cited. 